\title{
LANDSAT 9 THERMAL INFRARED SENSOR 2 SUBSYSTEM-LEVEL SPECTRAL TEST RESULTS
}

\author{
Boryana Efremova ${ }^{1}$, Aaron J. Pearlman ${ }^{1}$, Joel McCorkel ${ }^{2}$, Matthew Montanaro ${ }^{3}$ \\ Michael Hickey ${ }^{2}$, Allen Lunsford ${ }^{4}$, and Dennis Reuter ${ }^{2}$ \\ ${ }^{1}$ GeoThinkTank LLC, Washington DC 20009, USA \\ 2 NASA Goddard Space Flight Center, Greenbelt, MD 20771, USA \\ ${ }^{3}$ Rochester Institute of Technology, Rochester, NY 14623, USA \\ ${ }^{4}$ Catholic University of America, Washington DC 20064, USA
}

\begin{abstract}
Results from the Thermal Infrared Sensor 2 (TIRS-2) prelaunch spectral characterization at telescope and detector subsystem level are presented. The derived relative spectral response (RSR) shape is expected to be very similar to the instrument-level spectral response and provides an initial estimate of the RSR and its differences to the component-level RSR measurements. Such differences were observed at TIRS1 and are likely a result of angular dependence of the spectral response of the detector. The subsystem RSR measurements also provide an opportunity for a preliminary assessment of the spectral requirements. Final requirements verification will be performed at future thermal vacuum environmental testing with the fully assembled TIRS-2 instrument.
\end{abstract}

Index Terms - Landsat 9, TIRS-2, prelaunch characterization, spectral characterization, calibration

\section{INTRODUCTION}

The Thermal Infrared Sensor 2 (TIRS-2), scheduled for launch in December 2020 on board Landsat 9, is a pushbroom thermal imager with two spectral channels centered at $10.8 \mu \mathrm{m}$ and $12 \mu \mathrm{m}$. It is built at NASA Goddard Space Flight Center improving upon the Landsat 8 TIRS [1] design.

The instrument consists of a scene select mirror, followed by a four-element refracting F/1.64 telescope forming an image onto the focal plane array (FPA) consisting of three Quantum Well Infrared Photodetector (QWIP) [2] Sensor Chip Assemblies (SCA), filtered in the two spectral bands - $10.8 \mu \mathrm{m}$ (B10) and $12 \mu \mathrm{m}$ (B12) - by interference filters tailored to satisfy the spectral response requirements of each band. The three SCAs (SCA-A, B, and C) have $640 \times 512$ pixels each, of which only one effective row (the pixels from two rows are combined in one) per channel is used in science mode to observe the earth scenes. There is some overlap between the three SCAs, so the total number of detectors observing the earth is 1850 .
Knowledge of the relative spectral response (RSR) is required to ensure accurate radiometric calibration of the instrument, as the calibrating source (a blackbody at various temperatures) spectrum is averaged over the instrument RSR when deriving the calibration coefficients of the system [3]. On-orbit detector-to-detector RSR differences are manifested as striping in the imagery, and their characterization prelaunch can be used to improve the image quality and reduce the radiometric uncertainty. A number of requirements are placed on the spectral response shape and its uniformity along the row of 1850 detectors which span the width of the TIRS-2 15 degree $(\approx 185 \mathrm{~km})$ image (see e.g. [1] or [3] for details).

The instrument-level spectral response characterization test aims to characterize the RSR of the full instrument scene select mirror, telescope optics, filters, and detectors - in flight-like conditions and verify the compliance to the instrument requirements. In this paper we present the results from the TIRS-2 Imaging Performance and Cryoshell Evaluation (TIPCE) test campaign, concluded in March 2018 (see [4] for a detailed description). TIPCE was designed to evaluate the thermal performance of the instrument, characterize optical stray light effects, characterize the optical focus of the telescope, and perform a preliminary characterization of the spatial and spectral performance. The TIPCE configuration includes the major instrument components (flight FPA, filters, and telescope), but not the full instrument (e.g. it does not include the scene select mirror). Thus the spectral characterization is considered preliminary (subsystem level), but should provide a reliable first estimate of the instrument-level RSR and the accuracy with which it can be measured.

This paper is organized as follows. In Section 2 we describe the spectral response characterization test; in Section 3 the data processing is described; the results are discussed and compared to component-level measurements in Section 4. A summary is given in Section 5. 


\section{SPECTRAL CHARACTERIZATION TEST DESCRIPTION}

TIRS-2 spectral response measurements are performed, similarly to TIRS-1, using a blackbody (BB) at $1000 \mathrm{~K}$ as a source filling the entrance slit of a monochromator $(50 \mathrm{l} / \mathrm{mm}$ grating with blaze wavelength of $12 \mu \mathrm{m}$, and reciprocal dispersion $78.3 \mathrm{~nm} / \mathrm{mm}$ ) operating outside the thermal vacuum (TVAC) chamber. The monochromator output is collimated and directed into the TVAC chamber through a $\mathrm{ZnSe}$ window, where it is directed into the TIRS-2 aperture by the Calibration Ground Support Equipment (CGSE) optics. Outside the TVAC chamber a flip mirror is used to redirect the monochromator beam to a $\mathrm{HgCdTe}$ detector used as a radiance monitor.

The monochromator slit illuminates just a few $(\sim 10)$ of the 640 science row pixels per SCA which makes measuring the RSR of every individual pixel infeasible at subsystem and instrument level; instead several locations evenly distributed along the SCA are measured (at TIPCE three locations per SCA) in order to characterize any instrument-level spatial variations of the RSR shape. Component-level measurements indicate that the detector-to-detector RSR differences are small and within the instrument requirements. An example of the slit illumination pattern at one location on SCA-C is shown in the top panel of Figure 1.
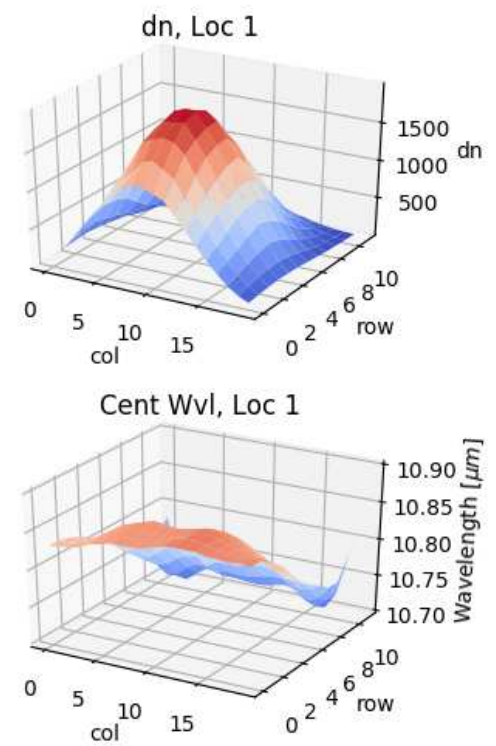

Fig. 1. An example of the slit illumination pattern (top panel) and the central wavelength of the derived RSR (bottom panel) at one location on SCA-C - the column numbers are relative to the location left edge at pixel 67.

During the spectral shape test the TIRS-2 instrument is commanded to read twelve rows for each spectral channel and each of the three SCAs comprising its focal plane array, as opposed to its science read-out mode when it is reading only two rows per channel. The 12-row reading pattern facilitates centering the slit image onto the science rows, and allows us to account for test artifacts observed over the slit image (e.g. effects from dispersion across the slit, defocusing, etc.).

\section{DATA COLLECTION AND PROCESSING}

The spectral measurements are collected to cover the in-band region for both spectral channels at $0.05 \mu \mathrm{m}$ wavelength step. For the $10.8 \mu \mathrm{m}$ channel (B10) 41 wavelength points are collected between 9.8 and $11.8 \mu \mathrm{m}$, and for the $12 \mu \mathrm{m}$ channel (B12) the sampled wavelength region is $11-13 \mu \mathrm{m}$ (again, 41 wavelength points are taken).

At each wavelength step TIRS- 2 collects 100 samples of the $\mathrm{BB}$, followed by 100 shuttered samples used to estimate and subtract the thermal background. The background subtracted TIRS-2 digital numbers (" $d n$ ") are then corrected using the $\mathrm{HgCdTe}$ reference detector measurements $\left(V_{r e f}\right)$ to account for the BB source spectral shape as well as any spectral dependence of the monochromator optics transmittances and other optical elements in the common optical path of the $\mathrm{HgCdTe}$ detector and TIRS-2. The spectral dependence of the chamber window and CGSE optics transmittances are not significant but are also accounted for $\left(\tau_{T I R S}\right.$ path $)$, as are the transmittances of optical components specific to the reference detector optical path $\left(\tau_{\text {ref path }}\right)$.

$$
d n_{\text {corr }}(\lambda, \text { pix })=\frac{d n_{\text {TIRS }}(\lambda, \text { pix }) \times \tau_{\text {ref path }}}{\tau_{\text {TIRS path }} \times V_{\text {ref }}} .
$$

Finally the RSR is constructed for each pixel of each location by normalizing the corrected $d n_{\text {corr }}$ values to their maximum:

$$
\operatorname{RSR}_{T I R S}(\lambda, p i x)=\frac{d n_{\text {corr }}(\lambda, p i x)}{\max _{\lambda}\left(d n_{\text {corr }}(\lambda, p i x)\right)} .
$$

The TIPCE data set contains individual RSR for all illuminated pixels of each location - a total of 9 locations per spectral channel (three locations for each SCA).

Several metrics were computed in order to compare the RSR for individual pixels and address the requirements. The central wavelength is computed as the midpoint between the $50 \%$ response points (band edges), the full width at half maximum (FWHM) is the distance between the $50 \%$ band edges, the signal-to-noise ratio (SNR) of the measurements is derived from the ratio of the magnitude of the $d n$ to the standard deviation of the $d n$, the in-band region is defined as the response above $1 \%$.

\section{RESULTS AND DISCUSSION}

Inspection of the per-pixel RSR shape for each location shows certain dependence of the RSR metrics on position along the 

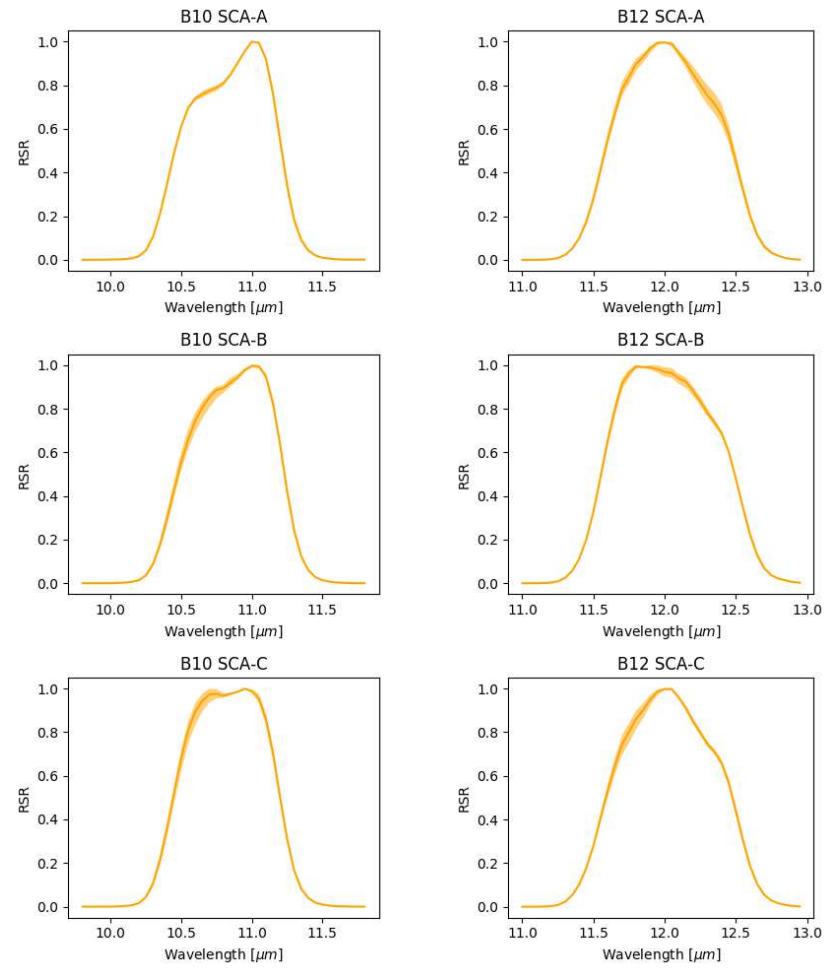

(a) B10.

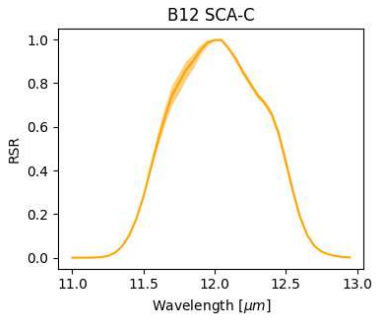

(b) B12.

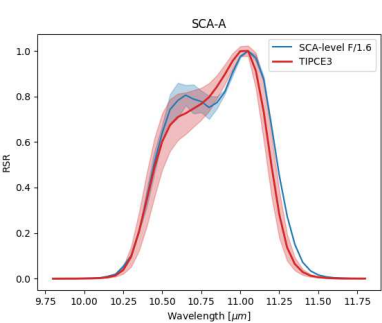

(a) B10

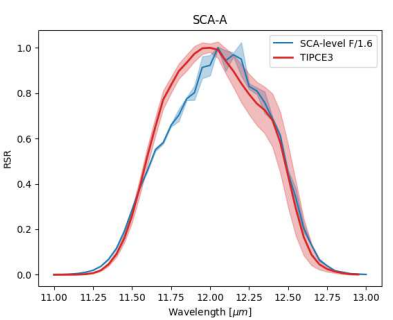

(b) B12

Fig. 3. Average RSR for SCA-A for the (a) $10.8 \mu \mathrm{m}$ and (b) $12 \mu \mathrm{m}$ channels. The subsystem-level RSR is shown in red; the roll-up of the component-level measurements is shown in blue. The shading represents the standard deviation of the RSR over all averaged pixels.

Fig. 2. Average RSR for each SCA for the (a) $10.8 \mu \mathrm{m}$ and (b) $12 \mu \mathrm{m}$ channels. The shading represents the $\mathrm{min} / \mathrm{max}$ envelope of the RSR over the three locations taken on each SCA.

slit image, which far exceeds the pixel-to-pixel RSR differences seen in component-level measurements, and is likely a test setup artifact rather than representing RSR differences intrinsic to the instrument. For example the center wavelength dependence on the position across the slit image indicates that we detect the dispersion across the monochromator slit (see the bottom panel of Fig. 1). Another feature of the per-pixel RSR at a given location is the gradual variation of the RSR shape with pixel position which is likely a combined effect of defocusing and angular dependence of the QWIP and filter response.

Thus we proceed to derive an average RSR for each of the observed locations instead of interpreting the per-pixel values. The average RSR per SCA (average over all pixels and all locations) is shown in Figure 2.

The RSR of the TIRS-2 instrument can also be derived by combining component-level measurements of the QWIP detector response and the transmittances of the filter and the optical components of the TIRS-2 telescope with optical modeling. The advantage of the component-level data is its availability for each pixel, so that it can be used for verification of the uniformity spectral requirements. The spectral response of the QWIP was characterized at the component level in the

NASA Detector Characterization Laboratory (DCL) at normal incidence and at F/4. In addition one SCA was measured at four different angles of incidence in order to sample the $\sim 17.5$ degree numerical aperture of TIRS-2 (F/1.64). As the DCL measurements show that the QWIP response has a strong dependence on the angle of incidence it is important to account for the F/\# difference between TIRS- 2 and the DCL measurements. To compare the component- to the subsystemlevel derived RSR a correction to the normal incidence QWIP response measurements was developed by weight-averaging the component-level angular measurements over the solid angle of the TIRS-2 aperture.

The component- to subsystem-level RSR measurements comparison is shown in Figure 3. The the subsystem level RSR is shown in red, and the roll-up of the component-level measurements (QWIP response, corrected for TIRS-2 F/1.64, combined with the filters and telescope optics transmittances) in blue. The agreement is within the standard deviation of the averaged subsystem-level RSR measurements, with the exception of the $11.7-12 \mu \mathrm{m}$ range for $\mathrm{B} 12$ where the instrument-level derived spectral response is higher. The agreement is much better than if the normal incidence DCL QWIP response measurements were used directly, thus validating the need of a correction to match the F/\#.

A preliminary assessment of the TIRS-2 spectral shape requirements is presented in Figure 4 (only a subset of the spectral requirements is shown as an example). The subsystemlevel derived RSR metrics are shown as circles, and, as many of the requirements are formulated for individual detectors, the component-level RSR derived results are also plotted as a solid line for all detectors. The subsystem-level measurements are largely in agreement with the component-level measurements within the errors with the exception of the B12 central wavelength and its blue edge which are about $0.02 \mu \mathrm{m}$ bluer in the subsystem-level measurements. 


\section{CONCLUSIONS}

The TIPCE test campaign provided an excellent opportunity to derive an initial estimate of the TIRS-2 RSR at subsystem level, and allowed us to perform a preliminary assessment of the spectral requirements. The TIPCE RSR results show good agreement with the component-level measurements after accounting for the angular dependence of the spectral response of the detector. Further improvements to the calibration equipment will be implemented to reduce the test artifacts that cause pixel-to-pixel RSR variability, and to decrease the measurement uncertainty.

Overall the TIRS-2 RSR measured at subsystem level shows compliance to the spectral requirements with few exceptions - consistent with the expectations based on componentlevel measurements.

The spectral test is part of a comprehensive pre-launch test plan to ensure that TIRS-2 will meet its performance and reliability requirements. The spectral characterization will be repeated at instrument-level TVAC which will test the completed instrument unit.

\section{REFERENCES}

[1] Dennis C. Reuter, Cathleen M. Richardson, Fernando A. Pellerano, James R. Irons, Richard G. Allen, Martha Anderson, Murzy D. Jhabvala, Allen W. Lunsford, Matthew Montanaro, Ramsey L. Smith, Zelalem Tesfaye, and Kurtis J. Thome, "The Thermal Infrared Sensor (TIRS) on Landsat 8: Design Overview and Pre-Launch Characterization," Remote Sensing, vol. 7, no. 1, pp. 1135-1153, 2015.

[2] M. Jhabvala, D. Reuter, K. Choi, C. Jhabvala, and M. Sundaram, "QWIP-based thermal infrared sensor for the Landsat Data Continuity Mission," Infrared Physics and Technology, vol. 52, pp. 424-429, Nov. 2009.

[3] Matthew Montanaro, Allen Lunsford, Zelalem Tesfaye, Brian Wenny, and Dennis Reuter, "Radiometric Calibration Methodology of the Landsat 8 Thermal Infrared Sensor," Remote Sensing, vol. 6, no. 9, pp. 8803-8821, 2014.

[4] Joel McCorkel, Matthew Montanaro, Boryana Efremova, Aaron Pearlman, Brian Wenny, Allen Lunsford, Amy Simon, Jason Hair, and Dennis Reuter, "Landsat 9 Thermal Infrared Sensor 2 Characterization Plan Overview," These Proceedings.
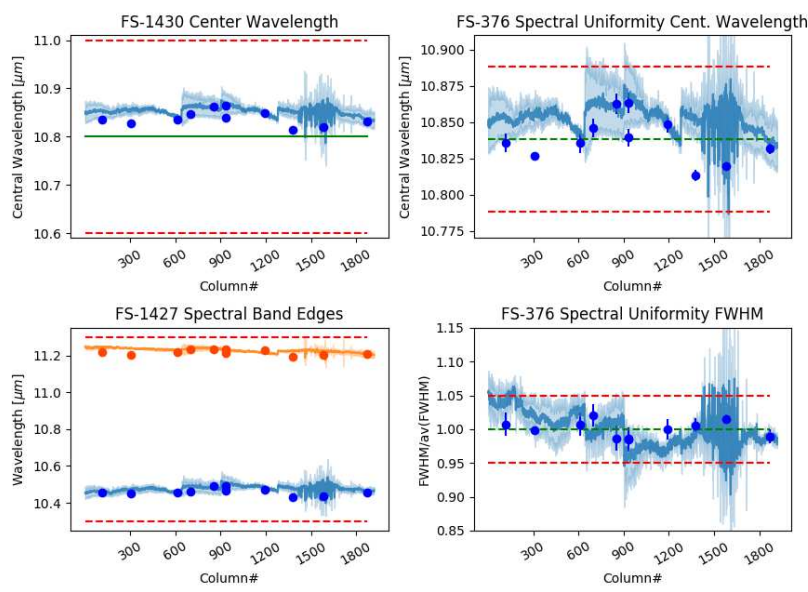

(a) B10
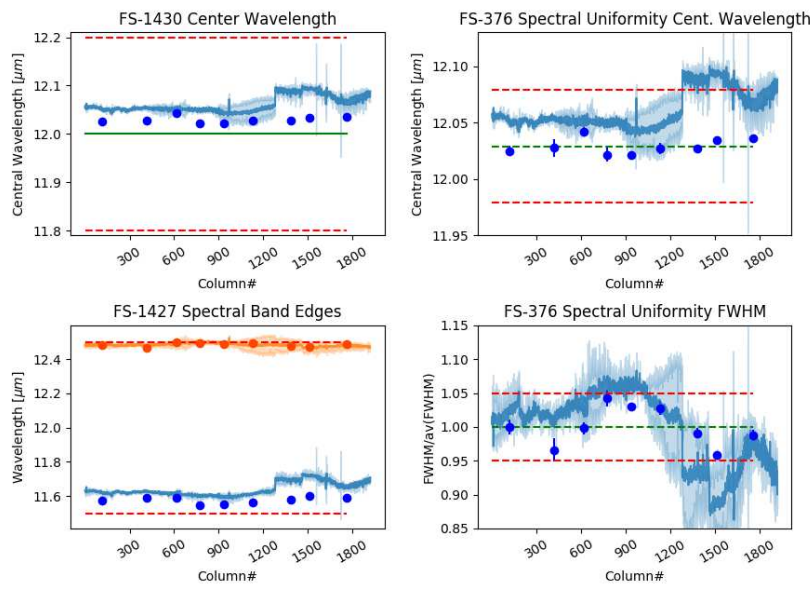

(b) B12

Fig. 4. Compliance to requirements examples for (a) B10, and (b) B12. The subsystem-level (TIPCE) results are shown as circles (the average for each location is shown). The component-level roll up is represented by solid lines, with the shading showing an uncertainty estimate based on the repeatability of two sets of measurements. The requirement limits are shown as red dashed lines. 